\title{
Entre dentelle et fragment : évolution de la tactique du vide rimbaldienne de la Saison aux Illuminations
}

\section{Avant-Propos à l'analyse des Illuminations}

À la différence d'Une saison en enfer, le corpus que constitue les Illuminations demande à tout analyste de faire montre d'une certaine minutie critique qui ne saurait être évitée, minutie que l'on pourrait presque qualifier de mise en garde. Ainsi, c'est sous le signe de la difficile unité critique ${ }^{1}$ et sous l'égide de deux thèses, celles de Bouillane de Lacoste (1949) et d'André Guyaux (1981), que s'inscrivent les Illuminations dans le corpus rimbaldien. Le recueil, factice ${ }^{2}$, s'établit comme un " fragment ${ }^{3}$. Une spécificité qui crée également toute la complexité et la difficulté d'analyse dudit corpus. Comme le souligne Steve Murphy (2009 : 443), il s'agit soit de respecter l'éclatement de l'œuvre en analysant chaque poème de façon indépendante soit de rechercher des thématiques communes, des liens même fragiles, qui uniraient, de manière superficielle ou profonde, certains poèmes de ce recueil artificiel. Cette deuxième possibilité, plus ardue, est également décriée par ceux pour qui toute tentative d'unité s'illustre comme une possible corruption de lecture des Illuminations. Peut-être est-il, ici,

Rafika Hammoudi - chercheur indépendant, titulaire d'un doctorat en Littérature française de l'Université Rennes 2 (La religion de Rimbaud) et titulaire d'une Maîtrise en LLCE Anglais de l'Universite de Tours (The Country of the Poet in Yeat's earlier poetry: The Crossways and The Rose). Adresse pour correspondance : rafika.hammoudi@gmail.com

1. En 1949, Bouillane de Lacoste établissait les Illuminations comme un texte postérieur à la Saison. La critique moderne propose une possible simultanéité, ou superposition partielle, des deux recueils. Ainsi l'Euvre-vie propose-t-elle une présentation de l'œuvre rimbaldienne dans laquelle les Illuminations précèdent la Saison. Pour justifier cette originalité éditoriale, Giusto note une " thèse pourtant contestable " (p. 1158). Steve Murphy, dans l'article qu'il consacre à ces deux thèses ("Les Illuminations manuscrites ", p. 11) note que, de manière générale, la thèse de Bouillane de Lacoste ne saurait être révoquée et que la difficulté critique des Illuminations se situe peut-être ailleurs (désordre des feuillets).

2. C'est sous les supputations de Verlaine que l'idée du recueil ainsi que son titre ont vu le jour et grâce aux analyses philologiques de Bouillane de Lacoste que le recueil doit sa forme actuelle. Ces dernières ne sont pourtant pas indubitables et indiscutables et rien ne permet d'affirmer que d'autres analyses ne viendront pas, si ce n'est bouleverser, du moins altérer de telles thèses.

3. L’appellation est de Rimbaud dans sa lettre de mai 1873 (Laïtou) à son ami Delahaye. 
nécessaire de réaffirmer qu'une telle fragmentation n'est pas spécifique à Rimbaud et qu'elle répond à un réel besoin littéraire et propre à son époque ${ }^{4}$ de s'interroger sur cette notion de continuité/discontinuité de l'œuvre, et particulièrement de l'œuvre poétique. Mais également, d'une interrogation liée au paysage urbain (humain ou architectural) omniprésent dans ces Illuminations (Carrozza, 1996). Il faut donc comprendre que dans les Illuminations la fragmentation sert et illustre le propos urbain.

Une discontinuité qui ne réside pas seulement dans l'aspect bref et concis des textes, mais également dans une discontinuité interne à chaque poème, cette impression de parcelles manquantes qui viennent surajouter à l'hermétisme des textes (Guyaux, 1985 : 191). Un choix stylistique qui tend à rapprocher Les Illuminations de la Saison, une nouvelle fois, Rimbaud utilise ce que nous avons nommé dans notre thèse la tactique $d u$ vide. Vide visible à travers la confusion dans laquelle se trouve plongé le lecteur ${ }^{5}$ à l'achèvement de chaque poème. Il ne s'agit pas d'un vide visible, tangible, mais d'un vide intérieur, de textes qui s'amorcent et se désamorcent sans que le lecteur ne comprenne le mécanisme de leur fonctionnement (ibid.). De là naît une relation trouble entre lecteur et auteur : le premier devant son malaise de lecture se voit dans l'obligation de reconnaître son incapacité à comprendre toutes les nuances pour ne pas dire les secrets du texte ${ }^{6}$; le second imprime et insiste sur sa supériorité auctoriale absolue et quasi intemporelle : "J'ai seul la clef de cette parade sauvage " 7 . Cette incapacité, voire infériorité, est nécessaire et primordiale à toute analyse du recueil, puisqu'elle admet le manque du lecteur, non plus comme une défaillance, mais comme un élément structurel de ces poésies et de leur réception.

Pourtant, cette théorie du fragment soulève deux problèmes fortement différents qui amènent le lecteur/critique vers deux positions de lectures divergentes. La première verrait dans la fragmentation, à la manière d'un miroir brisé, le morcellement d'un élément premier originel, dont l'œuvre ne serait qu'un éclatement. La seconde position supposerait une fragmentation de fait, auto-suffisante et autocentrée. Chaque poème devrait alors être lu en circuit fermé, chacun se suffisant à lui-même ${ }^{8}$. Ainsi, une alternance des deux théories semble nécessaire à la lecture des Illuminations ${ }^{9}$,

\footnotetext{
4. Sur le sujet: Chol I. (dir.). 2004. Poétiques de la discontinuité de 1870 à nos jours.

5. Alors que dans la Saison, la première confusion touche le narrateur lui-même et se propage au lecteur.

6. Puisque le "fragment est une forme littéraire inaccomplie ou définie par l'inaccompli » (ibid. : 197).

7. Particulièrement dans le cas de la fin des poèmes qui constitue, comme l'indique Guyaux : « un trou d'air " par lequel « aimerait s'échapper le texte tout entier» (ibid.).

8. Guyaux note que chaque texte est marqué « d'une forte unité » (ibid. : 192).

9. La première parution des Illuminations dans La Vogue (1886) mêlait, à une partie des Illuminations (certains poèmes ne seront retrouvés qu'en 1885), des poèmes de 1872. Néanmoins, rapidement s'est posé le " problème de l'organisation du recueil » (Brunel, 2004 : 14). Ainsi se pose la question de l'ordre des poèmes et, tout particulièrement, de celui des deux pièces en vers libres : "Marine » et « Mouvement». Brunel classe en trois principes ces différentes tentatives éditoriales des Illuminations (alphabétique, agglomératif, philologique) et leur reconnaît à toutes de certaines lacunes liées à cette im-
} 
loin du simple amas de poèmes juxtaposés (Murphy, 2000 : 30). Car il existe dans les Illuminations, à l'image de la Saison, une importante densité significative et, parallèlement, critique.

\section{L'engagement littéraire du vers libéré et le défi spirituel du verset}

L'élément qui permet et admet un rapprochement formel flagrant entre la Saison et les Illuminations est celui d'une poésie, majoritairement en prose dans la Saison et intégralement dans le cadre des Illuminations. Il faut à cela superposer l'image que véhicule la prose poétique à cette époque : celle du vers libre ou, pour éviter toute confusion (cf. Murat, 2002), du vers libéré d'un quelconque carcan formel. Il s'agit d'une forme poétique qui devient le symbole d'une liberté retrouvée à l'égard de la littérature et des nombreuses normes régissant cette société moralisatrice du dix-neuvième siècle. Ainsi, Rimbaud a cherché un signifiant (un vers libéré) qui correspondrait au mieux à son signifié (une tentative de libération morale et sociale). Un message perçu par le lecteur non néophyte qui comprend qu'il y a déjà, dans ce choix formel, une revendication qui fera écho au texte poétique. La forme devient indice du contenu.

Dans la Saison, Rimbaud fait le choix de subordonner la versification à la prose, de l'enchaîner à cette dernière. On retrouve imbriqués, dans Alchimie $d u$ verbe, sept poèmes en vers qui se présentent comme des remémorations ${ }^{10}$ du passé artistique du poète-narrateur et prennent donc, en opposition à la prose poétique, une forme fixe à la manière du souvenir figé qu'ils sont devenus ${ }^{11}$. En outre, il ne peut être question pour Rimbaud de respecter des règles de forme, dans un texte où, les règles de fond, en particulier sociale et religieuse, sont mises à mal. Il lui faut «trouver une langue " et faire que cette dernière fasse sens par elle-même, qu'elle soit vectrice d'une revendication par-delà le texte même.

Dans une certaine mesure, les Illuminations ne font pas exception, il reste un recueil de l'engagement et exige l'utilisation de ce vers libéré. Ainsi porte-t-il en son sein des poèmes à caractère politique : "Solde ", " Guerre ", "Soir historique »; d'autres à caractère social : « Parade », « Ouvriers"; d'autres encore religieux : comme « Barbare » ou " Génie "; sans compter les nombreux poèmes consacrés à la ville,

possibilité intrinsèque de connaître le véritable classement rimbaldien (ibid. : 17-20). Dès lors proposet-il plus simplement un retour à la tradition éditoriale des Illuminations, en l'absence de réel classement pertinent (ibid. : 20). En revanche, Murphy lit du feuillet 1 à 24 un réel enchaînement tel que désiré par Rimbaud (2000:30).

10. Bandelier note que la Saison « est donnée comme mémoriale : l'œuvre est placée d’emblée sous le signe du souvenir » (1988: 18).

11. Malgré des retouches importantes entre certains de ces poèmes et leur alter-ego des Derniers Vers, Rimbaud ne choisit pas de les fondre à la prose environnante. 
lieu de vie et de réflexion : "Ville [1] », «Ville [2] », « les Ponts », "Scènes ». Mais il existe également, dans ces Illuminations, un certain nombre de poèmes ostensiblement difficiles d'accès et qui se veulent, parfois, musicalement les plus aboutis comme " Matinée d'ivresse » ou " Départ ». Ces poèmes, où se lit l'ombre du Voyant, appellent à une autre lecture stratégique du poème en prose, celle de sa proximité, pour ne pas dire promiscuité avec le verset, volonté rimbaldienne qui avait déjà été déclenchée, plus lisiblement, dans la Saison.

En affranchissant ses écrits de ce carcan formel et terriblement humain, Rimbaud élève sa prose vers des sonorités sibyllines où le sens prévaut sur la forme. Le lecteur est donc constamment à l'affût de prophéties, de vérités cachées dans la mesure où il a été éduqué à la lecture du verset (cf. Conort, 2007), c'est-à-dire d'une parole divine, non versifiée, stylistiquement libre, où le sens prévaut également sur la forme. Élément que souligne Michel Sandras qui reconnaît dans les poèmes en prose une autre écriture du verset ${ }^{12}$ : tournant littéraire où le poème souhaite revenir à ses capacités liturgiques et religieuses. Le poète, être inspiré, delphique, veut retrouver une parole non artificielle, non humanisée dans ses règles et ses formes, revenir à l'hermétique et l'énigmatique. La forme n'est plus une excuse et l'hermétisme se fait évidence, à l'opposé du vers qui entraîne chez le lecteur une position plus suspicieuse.

Mais le verset, c'est également la forme divine, la possibilité pour le poète de se comparer à cette écriture, de la concurrencer. Le désir de sacraliser son œuvre en encourageant une lecture religieuse du texte avec tout ce que cela implique d'exégèse et de réflexes hermétiques, voire herméneutiques, dans le cadre des Illuminations. Rappelons qu'au dos de certains brouillons de la Saison furent retrouvées ces « Proses en marge de l'Évangile » qui offrent, dans leur dissection des textes johanniques, une opportunité pour Rimbaud de décrypter certaines clés de l'influence biblique et donc de les copier : d'écrire à la manière du verset. Ce qui semble intéresser Rimbaud dans la Saison ou les Illuminations, c'est cette possibilité d'une manipulation en aval de son propre lectorat et donc, logiquement, d'une main mise totale, quasi-divine, sur sa propre œuvre. Dès lors, comme le souligne Jean Molino, il sera important de se rappeler que dans la Saison, mais également dans les Illuminations, « la réception fait partie de la signification $»^{13}$.

\section{Points en blancs et points en noirs : broderie et dentelle}

Les deux termes sont particulièrement complémentaires dans le cadre de la Saison ou des Illuminations. Ainsi, la broderie, faite de points brodés, inclut dans son exécution une matière de base, un "fond ", tandis que la dentelle se veut une création ex nihilo faite de points noués. Le sont deux procédés qui structurent la Saison et des

12. Sandras, "Lire le poème en prose ». In Carla Van der Bergh, p. 41.

13. Molino, «La signification d'Une saison en enfer. Problèmes de méthode ». In Guyaux (dir.), Dix études sur Une saison en enfer, p. 12. 
Illuminations et sont sources des multiples dissensions critiques : Rimbaud se nourrit de ses lectures, de son observation et créé après réfléxion une ouvre originale.

Rimbaud va broder sa prose en choisissant d'orner ou d'ajourer à travers la création de ces espaces vides, blancs de lecture, qu'il a pensé, de la même façon qu'il a pensé le texte écrit dans ces termes et ces nuances. On pourrait même aller plus loin en affirmant qu'il y a, dans la Saison notamment ${ }^{14}$, deux textes : un texte en blanc (non écrit) et un texte en noir (écrit), un texte lisible et un texte volontairement illisible, un texte sonore et un texte silencieux. Si « la vraie vie est absente ", il devient naturel de se demander si le vrai texte ne l'est pas également. Dès lors, le non-dit prévaut autant que le dit et laisse s'immiscer dans son espace vide cette immensité critique qui entoure la Saison.

Une immensité critique, qui existe également dans le cadre des Illuminations, mais se comporte différemment, puisque comme le souligne Alain Vaillant ${ }^{15}$, les Illuminations imposent au lecteur une vision herméneutique de ses textes. L'herméneutique évoque une volonté délibérée de Rimbaud de complexifier et d'opacifier le sens de ses textes ${ }^{16}$. Ainsi, toute écriture hermétique ${ }^{17}$ implique une lecture herméneutique (Vaillant, 1997 : 83), notamment dans le cas d'un recueil comme Les Illuminations, où " l'esthétique du blanc et de la fragmentation " (ibid. : 88) appelle à cette recherche, cette enquête pour décrypter le texte. Reste en suspens la raison pour laquelle le poète a consciemment voulu obscurcir son texte, le retirer du "système de communication publique " auquel il se devait, initialement, de faire partie (ibid. : 97). Il s'agirait de capter l'attention du lecteur, de le forcer, sous cet apparent hermétisme, à chercher la réalité du poème, sa profondeur et sa valeur idéologique ou sociale (ibid.). Or si l'on admet cette hypothèse dans le cas des Illuminations, l'imaginaire ne serait qu'un vernis ${ }^{18}$ sous lequel transparaît un réel engagement poétique rimbaldien, ancré dans sa modernité ${ }^{19}$. En somme, les blancs de lectures se veulent ici

14. Seul texte pour lequel la comparaison brouillon/texte définitif permet de saisir lisiblement cette volonté rimbaldienne.

15. Ainsi peut-on citer de manière non exhaustive : «Verba hermetica, Mallarmé, Rimbaud, Hugo » (dans Romantisme, p. 81-97) ; "Posséder la vérité dans une âme et un corps » : la morale énigmatique d'Une saison en enfer » (dans Yann Frémy (dir.), Énigmes d'Une saison en enfer, p. 17) ou "Principes d'herméneutique rimbaldienne : gloses en marge de Chant de guerre parisien » (dans Parade sauvage, hommage à Steve Murphy, p. 137-154).

16. «Tous les textes littéraires seraient, par nature, polysémiques ; mais certains le seraient malgré eux («les classiques »); les autres au contraire, auraient pris conscience de leur disponibilité sémantique (les « modernes»), $c f$. Vaillant, $1997: 82$.

17. Cette dernière serait selon A.Vaillant : « l'emploi volontaire de codes dont ni la tradition ni l'œuvre même ne fournit la clé au lecteur» (ibid. : 83).

18. Guyaux indique que « beaucoup de poèmes des Illuminations ne nomment pas leur objet. Ce ne sont pas pour autant des énoncés sans objet, comme on le dit parfois, mais des énoncés dont l’objet n'est pas nommé, et qui ne rendent celui-ci que plus présent » (1991:53).

19. Guyaux évoque " un présent composé ", image d'un temps à "l'allongement inaccessible " (1985: 224-225). 
intangibles, invisibles, liés à une réflexion face à des textes qui résistent à toute [com] préhension immédiate.

\section{Lire les textes avec et sans modèles : la forme de l'informe}

En faisant le choix d'introduire la Saison comme « quelques hideux feuillets » qu'il " détache » de son "carnet de damné », Rimbaud annonce que le lecteur n'aura pas accès à l'intégralité de l'œuvre poétique ${ }^{20}$. Elle apparaît comme fragmentaire et aléatoire; il impose à l'esprit du lecteur une œuvre faite d'intermittences, que les titres et sous-titres vont renforcer. Mais il est également question d'insérer le pseudo-texte originel comme un ouvrage mythique qui hante le texte réel et surajoute à son hermétisme et son mystère. Rimbaud cherche à mythifier son œuvre, à retenir l'attention d'un lecteur contemporain friand de témoignage réel ou non (autobiographies factices, carnets de voyage...). Il retient ainsi l'une des leçons majeures de sa lecture biblique, celle de créer un constant qui-vive et une réception critique a posteriori de l'œuvre.

On retrouve une tactique similaire dans les Illuminations, à travers l'utilisation de la devinette ( Dévotion », « $\mathrm{H}$ », « Parade »), mais également du conte, de la légende ("Conte ", «Barbare ", " Royauté ») ou de tout autre procédé qui viendrait flouter les contours d'une situation d'énonciation trop précise. Il s'agit pour Rimbaud d'entremêler les espace-temps, d'ancrer le texte dans une réalité qui ne soit jamais formellement discernable. Même lorsque des noms de lieux sont évoqués comme dans les poèmes «Villes », il s'agit d'un cheminement d'idée chez le poète, d'un processus créatif proche, dans une certaine mesure, de l'écriture automatique : les transitions sont gommées et s'effacent au profit d'une unité de pensées plurielles, laissant le lecteur dubitatif, en proie à l'hermétisme de l'œuvre ${ }^{21}$. Ainsi, la ville se fait spatialement et temporellement étrange et étrangère ${ }^{22}$, presque déjà mythique, par l'héritage que son existence présuppose. Mais c'est également le spectacle humain offert à ses regards («Fêtes d'hiver », « Parade », «Scènes », « Les Ouvriers »...) qui permet au lecteur d'entrer aisément dans ces tableaux de vie à travers l'immuabilité humaine, dans ces habitudes et ses particularités, sans pour autant cantonner ces descriptions à une situation donnée. Immuabilité qui n'est en aucun cas synonyme de paralysie, de pétrification. Le mouvement reste un élément prépondérant aux Illuminations à travers

20. Bandelier considère ce terme comme une référence au journal intime, dont les feuilles mélangées créent l'absence de toute écriture chronologique dans la Saison - "discontinuité systématique de l'écriture» (1988:20-21).

21. Poème ambigu dans sa "genèse urbaine ", mais également dans sa " création » (Brunel, $2004: 311)$.

22. Ainsi, Buvik indique : « les mythes et les grandes créations de l'histoire de l'art et de la littérature sont très librement interprétés et exploités par Rimbaud, qui va jusqu’à les dénaturer complètement » (1988: 80). 
cette idée de changement, d'instabilité, de déplacement volontaire ou involontaire qui hante le corpus ( "Départ », "Vagabonds » et " Mouvement»).

Rimbaud semble tester, jauger la capacité de réflexion de son lecteur, puis récompenser le plus hardi, dans ce sentiment de détenir un secret non lisible de tous ( "Dévotion»), tout en multipliant les contradictions : hermétisme et simplicité. Il s’agit une nouvelle fois de bannir cette vision manichéenne du monde en proposant un " changement de perspective " (Brunel, 1999 : 903) constant qui vient éveiller, pour ne pas dire réveiller, le lecteur à ces nouvelles réalités, dans un instant visuel et non plus intellectuel. Ainsi, en choisissant de revenir à l'imagerie pure dans « Mystique », Rimbaud s'affilie à l'art liturgique traditionnel, comprenant qu'elle propose, par la vue, une compréhension rapide de la situation même pour le lecteur le plus naif et le moins instruit ${ }^{23}$. Il réaffirme son amour d'un art primitif, énoncé dans «Alchimie du verbe », dans une forme qui semble dépasser le mot. Il répond à la multiplicité des lecteurs, à la multiplicité de leurs approches, de leurs sensibilités à travers l'élaboration d'une pluralité des sens de lectures, d'analyses et de réceptions, plus ou moins complexes. Les saveurs se mêlent, se lient, se marient, sans jamais se rejeter ou se nier.

Dès lors, un peu à l'image de « Phrases », Rimbaud tend dans les Illuminations des cordes, des guirlandes, des chaînes d'or à des clochers, des fenêtres, des étoiles, puis les fait disparaître. Laissant le sentiment d'une tension, d'un lien, qui a existé mais qui s'est effacé, et qu'il laisse au lecteur le soin de retrouver dans une harmonie de dissensions critiques. Par ce stratagème, il poursuit la quête de la Saison, et du Voyant, mais la sublime en détruisant le gabarit qui fut le sien. Il ne reste de ce modèle premier que des fragments, dispersés dans l'œuvre, une multitude d'éléments, d'indices laissés par l'auteur, afin de guider le lecteur-critique et sa lecture sur la voie qu'il souhaite lui faire prendre, si le but de ce dernier est réellement de décrypter le poème et non de se laisser simplement bercer à son contact. L'œuvre ne devient jamais réellement indépendante de la volonté de son auteur.

\section{Et le lecteur combla le vide...}

Pour comprendre une telle vision de l'œuvre rimbaldienne, il faut revenir à la stratégie qui était celle de Rimbaud dans la Saison, à cette vivisection de l'écrit rimbaldien que Brunel évoque comme « une parole oraculaire » (Brunel, 1999: 403): parole non achevée, laissée hésitante entre son énonciation partielle et sa retenue, partielle également. Il est possible d'y inclure les spécificités d'une langue commune, non savante ${ }^{24}$.

23. Sacchi évoque un poème qui ne fait que «tendre vers cet instant tout à fait personnel d'une bizarre plénitude extatique » (2002: 199).

24. Si l'on se place du point de vue du narrateur de la Saison et de son impact sur le lecteur, il s'agit alors d'un langage vernaculaire, puisqu'il tente de se faire l'écho réaliste d'un individu pris au piège de sa propre destinée. 
Le narrateur poétique rimbaldien est victime d'une expérience qui le dépasse, lui qui a toujours été de "race inférieure " dans la Saison, ou qui a " foi au poison » dans "Matinée d'ivresse ». Il se retrouve dans une impossibilité de s'exprimer, de trouver des mots face à une expérience poétique qui le submerge, et présente cette parole sibylline, cette parole de contradiction comme un choix involontaire, imposé par les circonstances de l'expérience poétique.

Par ce fait, il marque l'action poétique qui donne l'impression d'une écriture qui se crée à l'instant même de sa réalisation et non ultérieurement, d'une écriture à vif, non retouchée, non ciselée. Dès lors, est-il donné conscience au lecteur qu'il lit un texte en creux où les propos énoncés ne sont que le contenant d'un vide à jamais perdu au cours de l'expérience. Il devient le récipiendaire d'un dépôt, d'une preuve de la véracité de cette expérience du narrateur, mais également un témoin privilégié de ce dernier. C'est sur ce point que la Saison profite le plus de l'impact du non-dit, parole laissée en suspens et que le lecteur peut combler à sa guise, lisiblement, visiblement, à la différence des Illuminations où le vide se veut plus implicite, tacite et ressenti.

Rimbaud instaure un sentiment élitiste chez cet autre-confident. Chaque lecteur se sent dépositaire d'une parole qu'il peut faire sienne, mais également de la véritable parole du narrateur. Combler ce vide, c'est devenir en quelque sorte le narrateur ou se faire le véritable dépositaire de l'œuvre, le véritable double du poète et donc son seul et unique lecteur véritable. Il offre à son lecteur le sentiment d'une œuvre unique, mais également d'une œuvre qu'il peut uniquement comprendre, utilisant cette vanité pour l'empêcher de réaliser qu'il est de nouveau victime d'une tactique poétique.

Ainsi, Rimbaud manipule ce futur lecteur, lui donnant l'illusion d'une œuvre en creux, là où, en réalité, il ne s'est absenté pas un seul instant ${ }^{25}$. Tout vide de l'œuvre est un vide volontaire, réfléchi, empli de la présence du poète. Ce qui permet de se demander si, en réalité, la Saison ou les Illuminations offrent réellement un interstice où s'immiscer, si l'œuvre n'est pas saturée par cette omniprésence du poète. Dès lors, il nous paraît évident qu'en 1873, à la rédaction de la Saison, Rimbaud échafaude la construction d'un auteur-poète transcendant la mort et le temps, en choisissant de s'infiltrer dans toutes les aspérités de son œuvre ${ }^{26}$. Tactique $d u$ vide qu'il développera, différemment, plus subtilement, peut-être même plus méticuleusement dans les Illuminations, dans l'élaboration de textes qui se veulent avant tout l'illustration d'une créativité constante, d'un renouvellement ininterrompu, non pas seulement de la création poétique, mais aussi du monde du poète. Il s'agit de proposer à la lecture des superpositions de sens, échos des immenses structures qui peuplent le re-

25. "Au cours de l'été, le texte a sans doute été rédigé dans la rage. Mais l'ouvrage a été très travaillé » (Brunel, 1999 : 401).

26. " Au centre, un narrateur, ou du moins un locuteur. [..] En tout cas un moi qui s'affirme constamment, même s'il lui arrive de céder la parole à l'autre »(Brunel, 1999 : 1193). Autre qui se veut également un autre moi. 
cueil. L'hermétisme de l'œuvre lui permet cette double approche : en dedans et en dehors du monde. Rimbaud est simultanément créateur et lecteur de l'œuvre, mais jamais un simple fantôme.

Dès lors en arrive-t-on à se demander, à la lecture de ces deux textes, si Rimbaud ne crée pas un lecteur à son image. Un lecteur qui viendra compléter l'unité si recherchée par toute œuvre, mais qui ne sera vraisemblablement qu'un autre lui-même. Compléter cette œuvre et ces blancs reviendrait à parachever l'œuvre et à prendre part à l'entreprise poétique, même si la démarche ne se veut pas réellement autonome, mais guidée par un poète presque transcendant. En acceptant ce pacte, le lecteur flirte avec l'agréable impression d’être parfois lui-même poète.

\section{BIBLIOGRAPHIE}

Bandelier D. 1988. Se dire et se taire : l'écriture d'Une saison en enfer d'Arthur Rimbaud. Neuchâtel. À la Baconnière.

Bouillane de Lacoste H. de. 1949. Rimbaud et le problème des Illuminations. Paris. Mercure de France.

Brunel P. 1983. Arthur Rimbaud ou L'éclatant désastre. Seyssel. Champ Vallon.

Brunel P. 1999. Rimbaud. Euvres complètes : poésie, prose et correspondance. Paris. La librairie générale française.

Brunel P. 2004. Éclats de la violence : pour une lecture comparatiste des "Illuminations" d'Arthur Rimbaud. Paris. José Corti.

Buvik P. 1988. «Les villes de Rimbaud. Poésies et thématique des descriptions urbaines dans les Illuminations ». Parade sauvage $\mathrm{n}^{\circ}$ 5. 76-87.

Carrozza M. L. 1996. «Paysage urbain : matérialité et représentation ». Les Cahiers du Centre de Recherches Historiques $\mathrm{n}^{\circ} 17$. En ligne : http://journals.openedition.org/ccrh/2600.

Chol I. (dir.). 2004. Poétiques de la discontinuité de 1870 à nos jours. Clermont-Ferrand. Presses universitaires Blaise Pascal.

Conort B. 2007. «Si verset il y a.. ». Études littéraires. Vol. 39 n¹. 141-147.

Frémy Y. (dir.). 2014. «Énigmes d’une saison en enfer ». Revue des sciences humaines n³13.

Giusto J-P. 1980. Rimbaud créateur. Paris. Presses universitaires de France.

Guyaux A. 1985. Poétique du fragment : essai sur les "Illuminations » de Rimbaud. Neuchâtel. À la Baconnière.

Guyaux A. (dir.). 1991. Dix études sur Une saison en enfer : actes de la journée du Colloque du centenaire, Marseille, 9 novembre 1991. Neuchâtel. À la Baconnière.

Guyaux A. 1991. Duplicités de Rimbaud. Paris-Genève. Honoré Champion-Slatkine.

Rimbaud A. 1991. Euvre-vie, édition du centenaire. Paris. Éd. A. Borer. Arléa.

Rimbaud A. 1999. Euvres complètes : poésie, prose et correspondance. Paris. P. Brunel (éd.). La librairie générale française.

Murat M. 2002. L'art de Rimbaud. Paris. José Corti.

Murphy S. 2000. «Les Illuminations manuscrites ». Histoires littéraires nº 1 . En ligne : http:// histoires-litteraires.fr/les-illuminations-manuscrites/.

Murphy S. 2009. Stratégies de Rimbaud. Paris. Honoré Champion. 
Premuda Perosa M. L. 1988. Une écriture de l’énigme : "H» de Rimbaud. Napoli. Edizioni Scientifiche Italiane.

Sacchi S. 2002. Études sur les Illuminations de Rimbaud. Paris. Presses de l'Université de ParisSorbonne.

Vaillant A. 1997. «Verba hermetica, Mallarmé, Rimbaud, Hugo ». Romantisme n95. 81-97.

Vaillant A. 2008. "Principes d'herméneutique rimbaldienne : gloses en marge de Chant de guerre parisien ». Parade sauvage (hors-série hommage à Steve Murphy). 137-154.

Van der Bergh C. 2007. «Le rôle du verset lors de la transition du grand poème au petit poème en prose, dans les années 1830-1840 en France : pertes et profits ». Études littéraires. Vol. $39 \mathrm{n}^{\circ} 1.25-41$.

\section{Between Lace and Fragment: an evolution of Rimbaud's Tactic of Emptiness from $A$ Season in Hell to Illuminations}

ABSTRACT: A Season in Hell and Illuminations both of these collections are considered as masterpieces of Rimbaud's poetry. Whereas A Season in Hell is presented as his artistic testimony, Illuminations, on the other hand, is considered by critics as an abstruse writing, an enigma. Acknowledging critical scholarship on this topic, as well as inherent differences of both collections, this article will show that a link could be found between these two works. This thread, which creates what we would like to call Rimbaud's embroidery, was created by himself through silence and words. That is this will of Rimbaud for both works and the readers that we will discuss here, a strategy that we have named: Tactic of Emptiness.

Keywords: Arthur Rimbaud, French poetry, 19th century French literature, A Season in Hell, Illuminations, tactic of emptiness. 